\title{
Adapting Clinical Skills to Telehealth: Applications of Affirmative Cognitive-Behavioral Therapy with LGBTQ+ Youth
}

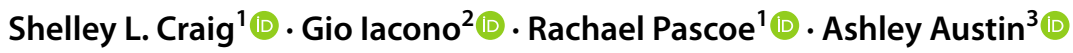 \\ Accepted: 2 February 2021 / Published online: 2 March 2021 \\ (c) The Author(s), under exclusive licence to Springer Science+Business Media, LLC part of Springer Nature 2021
}

\begin{abstract}
Online social work services (e.g., telemental health; telebehavioral health; virtual care; telehealth) present significant opportunities for clinical social workers to provide effective care to marginalized populations, such as LGBTQ+ youth. The COVID-19 pandemic has led to an increased focus on telehealth, and while there are excellent resources to guide ethics, standards, and legal decisions (NASW, n.d.), there is less guidance in the literature to specifically inform the adaptation of offline clinical skills to telehealth, particularly for LGBTQ+ youth. To address this gap, we present examples from our experience offering AFFIRM, an affirmative cognitive behavioral therapy (CBT) group intervention now being delivered through telehealth. Specifically, this paper will: (a) describe the key considerations for the delivery of CBT via telehealth to youth; (b) detail specific clinical skills and strategies to enable successful online implementation; (c) describe the adaptation approach through a case study of cognitive restructuring with a transgender youth; (d) and offer specific guidance to support clinicians to adapt their clinical skills to the virtual environment.
\end{abstract}

Keywords LGBTQ+ youth $\cdot$ Telehealth $\cdot$ Affirmative practice $\cdot$ Cognitive behavioral therapy $\cdot$ Mental health

Telehealth has been defined as the "remote provision of healthcare services using technology to exchange information for the diagnosis, treatment, and prevention of disease" (Kaiser Family Foundation 2020, para 2). Telehealth, regarded as a critical modality to support marginalized and underserved populations (Camhi et al. 2020), provides significant opportunities for clinical innovation (Kahn 2015). Marginalized populations, such as LGBTQ+ (e.g., lesbian, gay, bisexual, transgender, queer, questioning) youth, may be a population that most benefits from virtual care given their well-documented mental health disparities and use of information communication technologies (ICTs) to cultivate social support and build resilience (Craig et al. 2015, 2020;

Gio Iacono

gio.iacono@uconn.edu

1 Factor-Inwentash Faculty of Social Work, University of Toronto, 246 Bloor Street West, Toronto, ON M5S 1V4, Canada

2 School of Social Work, University of Connecticut, 38 Prospect Street, Hartford, CT 06103-2814, USA

3 Ellen Whiteside McDonnell School of Social Work, Barry University, 11300 NE 2nd Avenue, Miami Shores, FL 33161-6695, USA
McInroy and Craig 2018). LGBTQ+ youth continue to be among the most disadvantaged populations globally compared to their straight and cisgender counterparts (EGALE 2020; Human Right Campaign Foundation 2020. LGBTQ+ youth face disproportionate discrimination in multiple domains, such as a lack of employment and educational opportunities, barriers to essential health and mental health services, higher rates of homelessness, social isolation, and family rejection that contribute to increased suicidality, depression and other mental health vulnerabilities (EGALE 2020; Fowlkes 2020; Russell and Fish 2016; Taylor et al. 2011; True Colors United 2020; Whittington et al. 2020).

Minority stress theory provides an empirically supported theoretical framework for understanding the issues LGBTQ+ youth experience (Meyer 2003). According to minority stress theory, LGBTQ+ populations experience health and mental health disparities due to distinct anti- LGBTQ+ stressors and discrimination that are perpetuated through structural and interpersonal stigma. Anxiety, depression, suicidal ideation, and suicide attempts are disproportionately reported in the LGBTQ+ population as a result of minority stress (Burton et al. 2013; Borgogna et al. 2019). Furthermore, these disparities may be complicated by LGBTQ+ youth's multiple and intersecting forms of marginalized identities 
and status (e.g., racial, socioeconomic, cultural, religious, etc.), which may contribute to unhealthy coping strategies such as substance misuse, negative thinking and worry, and risky sexual behaviors (Craig et al. 2012; Hatzenbuehler 2009; Russell and Fish 2016). For instance, LGBTQ+ youth have reported greater ruminative thinking styles, emotional suppression, and psychological distress while experiencing minority stress (Hatzenbuehler et al. 2008). LGBTQ+ youth have also reported blunted emotional regulation and awareness compared to their non- LGBTQ+ youth counterparts (Hatzenbuehler et al. 2008; Russell and Fish 2016). Given the universality of these experiences for many LGBTQ+ youth, affirmative clinical support which directly targets the impact of minority stress is warranted (Austin and Craig 2015; EGALE 2020). The COVID-19 pandemic has further impacted LGBTQ+ youth by mandating a quarantine in family environments that may be unsafe and outright hostile (Fish, et al. 2020), which in turn may increase their risk of experiencing abuse, victimization and violence (Human Rights Campaign Foundation 2020). Specifically, lack of housing access, employment, and social services are exacerbated as a result of the COVID-19 pandemic for LGBTQ+ youth and emerging research has identified mental health challenges (Gibb et al. 2020). Despite these exacerbated stressors, the pandemic restrictions have reduced access to traditional offline services (Zhou et al. 2020), thus creating a demand for relevant virtual mental health care for LGBTQ+ youth (Fish et al. 2020). COVID-19 has demanded pragmatism and adaptability from social work clinicians to effectively deliver mental health care (Saidipour 2020). This paper describes the application of clinical skills to telehealth through the example of AFFIRM, an empirical intervention designed for LGBTQ+ youth.

Emerging research documents the benefits of online engagement to the wellbeing of LGBTQ+ youth, the critical importance of these activities to their mental health, and the potential receptivity this population would have to an online intervention. A major North American study with LGBTQ+ youth $(n=6309)$ found that participants (ages 14-29) are very active within a range of online communities and regularly utilize a variety of ICTs such as social media applications (Craig et al. 2017). McInroy and Craig (2018) found that LGBTQ+ youth engage in online communities to find support, resources, and information, which may be related to challenges in accessing adequate offline supports. Even in urban areas that have some offline support, LGBTQ+ youth consistently report that online communities are safer than offline settings (Craig and McInroy 2014). Additionally, online resources may facilitate identity exploration, cultivate resilience, and improve overall wellbeing (Craig and McInroy 2014; Craig et al. 2015). Thus, LGBTQ+ youth may already be primed to favourably respond to technology-mediated mental health interventions because of their comfort in finding community through ICTs.

\section{Cognitive Behavioral Therapy for Youth: Telehealth}

Cognitive behavioral therapy (CBT) has been considered a gold-standard mental health approach for young people experiencing a myriad of mental health issues (Craig et al. 2013; Silverman et al. 2008), and has been effectively delivered offline in a variety of clinical and community settings (Merrill et al. 2003; Scott et al. 2016; Simons et al. 2010). Numerous studies have supported the efficacy of CBT delivered through telehealth (TCBT) for a variety of mental health issues (e.g., depression and anxiety) for youth and adults (Andrews et al. 2010; Davies et al. 2014; Furmark et al. 2009; Khanna et al. 2007; Kiropoulos et al. 2008; Spence et al. 2011; Wright et al. 2017). For instance, a meta-analysis consisting of 22 randomized-control trials has shown that TCBT has significant effects for treating anxiety and depressive disorders (Andrews et al. 2010). Additionally, a recent systematic review of interventions for youth (up to 18 years) $(n=3113)$ diagnosed with anxiety and depression, found medium effect sizes for TCBT interventions compared to waitlist participants (Grist et al. 2019). Additionally, this study found that the degree of clinician support in TCBT directly impacted overall intervention effects with even minimal contact (e.g., active therapist engagement; support in applying therapeutic techniques; $>90 \mathrm{~min}$ of time), and produced larger effect sizes compared to self-help, self-paced CBT online modalities. Taken together, the extant literature supports the efficacy of TCBT for a variety of mental health challenges for youth (Hollis et al. 2017; Khanna et al. 2007; Poppelaars et al. 2016; Spence et al. 2011; Wright et al. 2017).

Telehealth-based CBT has been applied to LGBTQ+ populations through guided module-based interventions. For example, the program Rainbow SPARX, uses a game-based platform to deliver CBT skills and psychoeducation specifically for LGBTQ+ youth (Iacovides et al. 2017). Rainbow SPARX demonstrated a significant decrease in depression ( $p<0.0001$, pre- to post-effect size $d=1.01$ ) for sexual minority youth between ages 13 and 19 upon intervention completion, which was maintained at three-month follow up (Lucassen et al. 2015). The mobile phone online intervention HealthMpowerment was delivered to young black men who have sex with men and black transwomen with the aim of decreasing risk factors for HIV transmission (Hightow-Weidman et al. 2015). Despite a small sample size, the authors found significant decreases in social isolation $(p=0.050)$ and depression symptoms $(p=0.045)$ at study conclusion and follow up (Hightow-Weidman et al 2015). Other LGBTQ+ 
specific CBT-based interventions for LGBTQ+ youth have been offered in person (Bochicchio et al. 2020), or are not CBT-specific (Tasnim et al 2020), demonstrating that a gap exists in therapist-led LGBTQ+ specific CBT telehealth interventions.

\section{Intervention Example: Affirmative CBT}

As the first empirically-supported mental health intervention for LGBTQ+ youth, AFFIRM is a manualized affirmative, eight-session CBT intervention that has been found to reduce depression, sexual and mental health risks, and increase adaptive stress appraisal and coping skills in offline (Craig and Austin 2016) and online delivery (Craig et al. 2021). AFFIRM was initially designed to be delivered in a group format to youth with a range of intersectional identities (Craig et al. 2013). AFFIRM aims to: (a) acknowledge and validate intersectional minority stressors LGBTQ+ youth experience; (b) engage in an exploration of the various ways LGBTQ+ youth currently cope with intersectional anti- LGBTQ+ stressors; (c) explore and strengthen alternative cognitions and behaviors, and coping skills that affirm their identities; and (d) develop affirming support networks with peers and allies. AFFIRM sessions typically move through: (a) a warm up/review; (b) discussing session objectives; (c) a behavioral activity; (d) extensive practice and rehearsal; and (e) a group reflection and summary (the content of the AFFIRM modules is described in Craig and Austin 2016; Craig et al. 2019). AFFIRM incorporates CBT techniques and strategies (e.g., thought records, cognitive restructuring, behavioral activation and experiments) to help LGBTQ+ youth develop adaptive coping skills. AFFIRM teaches LGBTQ+ youth these skills through psychoeducation, reflection, and rehearsal (i.e., simulation of real-life experiences) within an affirmative framework that acknowledges and validates intersectional anti- LGBTQ+ stigma and discrimination. AFFIRM has been delivered offline utilizing an established protocol for implementation, including a comprehensive and standardized facilitator training, a facilitator intervention manual, and a participant workbook (Craig et al. 2020). All AFFIRM material has been developed and evaluated through a rigorous community stakeholder approach, e.g., working closely with LGBTQ+ youth, social work clinicians and academics, and community-based organizations (Austin and Craig 2015; Craig et al. 2019).

Given that the AFFIRM intervention has been found to be an effective clinical approach to improve the mental health of vulnerable LGBTQ+ youth offline (Craig and Austin 2016), and CBT has been an established modality for supporting the improvement of mental health for the general youth population both offline and online (Compton et al. 2004), it was quickly determined that AFFIRM would be adapted for online delivery to meet the service delivery needs of the COVID-19 environment. Clinical psychotherapy literature and practice policies from registration or licensure bodies provide some guidance for advancing telehealth (APA 2013; Backhaus et al 2012; CPA 2006; McCarty and Clancy 2002; NASW, n.d.; Reamer 2014), however, specific clinical social work strategies for the delivery of CBT to LGBTQ+ youth are limited. The following sections outline the critical components of affirmative CBT with LGBTQ+ youth and describe clinical approaches to address these key ingredients through telehealth. Further detailed examples are offered in Table 1.

\section{Key Clinical Considerations for the Telehealth Delivery of CBT}

\section{Setting the Stage: Adjusting Programs and Orienting Clients}

To address the needs of LGBTQ+ youth, an online offering of AFFIRM was designed to follow the same manualized curricula as the offline AFFIRM intervention (Craig and Austin 2016) and delivered in similar weekly two-hour telehealth sessions (including a 10-min break) plus a one hour meet and greet offered prior to the first session. AFFIRM Online was facilitated by social work clinicians (with a range of sexual, gender, and racial identities, and between 7 and 15 years of clinical experience) who were trained in delivering offline AFFIRM groups. During the transition to online, AFFIRM clinicians also received extra individual clinical training and weekly peer supervision to support the effective delivery in this new format. This support enabled skill development among all facilitators, as well as the generation of clinical adaptations that are detailed in the following sections.

Following consultation with the regulatory social work organization, the authors decided to offer AFFIRM online to youth via Zoom, as this was a familiar and preferred platform for youth participants. As well, CBT activities were adaptable to the online format through Zoom's user-friendly interface. To support youth engagement, detailed Zoom instructions were provided through email prior to the beginning of group, and a brief tutorial was offered during initial sessions (i.e., AFFIRM meet and greet), which allowed participants to increase feelings of comfort and safety with the intervention, the facilitators, and interact on the Zoom platform prior to the first session. The initial meeting allowed for any technological troubleshooting. Early group sessions dedicated some group time to instructing members on the Zoom platform, how to interact online (by taking turns speaking, muting microphones when not speaking), how to personalize their names (e.g., adding pronouns), and how to 
Table 1 AFFIRM online adaptations

Online adaptations and clinical skill considerations

\section{Focus}

Setting the stage: beginnings
Therapeutic engagement
Develop competency in technology-mediated platforms (e.g., Zoom)

Take a moment at the beginning to do a technology check with clients (e.g., working camera, chat, audio) and troubleshoot any problems they may be having

Prompt participants to all turn on video/audio at the beginning of session to validate identity, assure confidentiality, and assess for crisis or emergencies

Provide instructions and a demonstration of Zoom functions (e.g., gallery versus speaker mode, utilizing chat, changing name, raising hand, etc.)

Adjust Zoom settings accordingly: enable chat box, enable emoji reactions, allow for breakout sessions for members in distress with a designated facilitator, enable waiting room)

Utilize account screen name as name tag (name/pronouns) - ensure clients remove sensitive information (e.g., pronouns) after session in case they are not out in other contexts that they may be attending meetings by Zoom

Ensure a norm is established regarding taking turns speaking (e.g., use of Zoom function to put "hand up," actually raising hand, etc.)

Regularly ask about accessibility needs for online participation

In a cofacilitation situation, determine which facilitator will be monitoring the chat. Address at the front end that the chat is to speak to everyone or facilitators, and not for private side conversations with other group members. Adjust Zoom settings to ensure private chatting is only enabled to the facilitators

Determine prior to the start of group who will support a group member individually in the event of an emergency or crisis while group is occurring

Ensure participants have crisis/emergency numbers-provide regularly at each session. Ensure participants are aware that the supports being offered are not 24/7 crisis services. Ensure facilitators have clients' current address and contact information in case of emergency

Transparency and therapeutic use of self: discuss understandable challenges with conducting CBT online, and potential client discomfort and general awkwardness in conducting therapy online. Aim to demonstrate extra therapeutic transparency and vulnerability as needed to join with, normalize and validate clients' stress during the pandemic

Adapt icebreaker activities as appropriate (e.g., use of Google Doc sharing)

Check in with clients potentially every few minutes, asking discussion questions to enhance engagement

Trauma-informed approach: acknowledge trauma and triggers during the COVID-19 pandemic and other global events; respond to identity-specific trauma

Aim for two check-in/reminder messages (email or text) in between sessions to enhance engagement and reduce attrition

Engage in between-session supports (e.g., light case management, providing resources; one-toone coaching)

Utilize PowerPoint slide deck for initial session to build structure and to ensure effective dissemination of information

Utilize grounding strategies (e.g., mindful awareness of senses) at the beginning and end of sessions as needed to support acclimating to an online environment and addressing stress and anxiety as a result of global events

Aim for more "share screen" time (which limits viewing all participants in gallery mode) at the beginning and early stages of the session. Decrease this over time as clients become more comfortable and familiar with each other

Ask for participants to send the clinician a chat message if they need to step away from the camera for a period of time (e.g., bathroom break, etc.). This may help keep clinicians aware of any potential trigger or crises

Clarify and ask for repeats (ask for clarification if members were hard to hear or unclear). Be upfront in reporting technology issues you may be having with the members and have a backup plan with your cofacilitator to account for any disruptions in the group as a result 
Table 1 (continued)

Online adaptations and clinical skill considerations

Facilitate engagement: view screen in gallery mode [not speaker mode] and encourage members to do the same, encourage engagement through prompting conversation, encourage member to member discussion, intersperse activities, discussions, and individual reflective work throughout the session

Encourage members to reach out to facilitators between sessions, and encourage members reaching out to bring their sharing with the facilitator back to the group

Accept and facilitate some member's self-selection out of the group and acknowledge attrition as the group continues. Online groups are not for everyone and alternative options can be problem solved with members

Cognitive restructuring approaches

Behavioral approaches

Use screen share function periodically throughout session to demonstrate the CBT triangle and worksheets

Modify scaling questions to focus on positive action, beliefs, and positive framing: from "On a scale of 1-10, 1 being "low" and 10 being "high", how stressed were you this week?" to "On a scale of 1-10, 1 being "low" and 10 being "high", which activities affirmed you and to what extent?"

Use Zoom white board and screen share to teach and conduct ABCD method and other approaches; send white board and group chat brainstorm ideas via email/text to youth

Use Zoom white board and screen share to teach and conduct ABCD method and other approaches; send white board and group chat brainstorm ideas via email/text to youth

Utilize electronic and fillable worksheets/workbook to successfully teach and practice cognitive restructuring strategies (e.g., ABCD method; thought stopping) directly on clients' computers or electronic mobile devices

Provide more practical examples (e.g., ABCD and thought stopping) to support clients in better grasping the approach in modifying unhelpful thoughts and negative core beliefs

Embed cognitive strategies and practices into each session, including more behaviorally-focused sessions, to ensure mastery of cognitive skills

Make use of the group chat on Zoom to share cognitive restructuring (e.g., disputing beliefs) outcomes to better grasp cognitive skills online

To support the acquisition of cognitive restructuring skills, include brief between-session individual support (e.g., emailing and texting additional examples, assigning journal writing to articulate thoughts and emotions, and brief phone coaching) for youth that continue to struggle to acquire skills

Increase use of, and practice, of grounding/calming strategies earlier in the intervention

Prepare for Hope Box (Craig and Austin 2016) early in the intervention by discussing and providing instructions (e.g., collect affirming symbolic objects for Hope Box; pick up art supplies and box to create Hope Box)

Encourage group members to engage in behavioural experiments-exposures and activation- in and out of group.

Address online barriers to affirming behaviors, particularly barriers related to prejudice and discrimination

Dedicate extra time and focus on finding online and physical distancing affirming activities (e.g., LGBTQ+ online groups and events), and adapting activities (e.g., exercise, social time, sports) that could be conducted individually or with safe social distancing

Adapt behavioral experiments and exposures (i.e., testing and challenging unhelpful cognitions through specific actions) to online platforms - collaboratively working with LGBTQ+ youth in setting up behavioral experiments and exposures online utilizing video (e.g., FaceTime, WhatsApp, Google Hangouts) or telephone

Prepare a behavior action plan with members in the week between groups (e.g. engage in one self-care activity over the week)

Developing affirming social support networks

Set up and encourage group members early on for continued contact after the group ends (Instagram messages, WhatsApp, Facebook, etc.)

Highlight importance of online community/activities during isolation 
Table 1 (continued)

Online adaptations and clinical skill considerations

Support community-building and affirmative social support networks earlier among participants via social media/online platforms outside of AFFIRM

Utilize LGBTQ+ youth strengths, collective knowledge and wisdom in sharing online resources and activities that support mental health

Endings: post-intervention

Follow up with youth post-intervention as needed

Create opportunities for AFFIRM graduates to engage online (Facebook Group; Instagram page)

Celebrate members' successes!

verbally participate or chat, which enabled the facilitators to begin to create a safe therapeutic environment.

\section{Therapeutic Engagement: Increase Clarity of the Intervention Process}

While CBT interventions are clear about the structure and course of treatment (Beck 1993), in an online treatment environment, there may be a greater need to provide additional transparency and clarity regarding the intervention process, the outline of the sessions, and clarification of expectations. This sense of enhanced transparency can be calming for anxious LGBTQ+ youth and may provide a sense of empowerment and ownership of their treatment process (Craig et al. 2012). For example, in contrast to the offline sessions, the authors developed a structured PowerPoint presentation for the initial session to provide participants a roadmap of the AFFIRM intervention. This presentation included the rationale for AFFIRM, additional details about each session, instructions for accessing the intervention material, and how to $\log$ into the Zoom meeting room. The social work clinicians delivering AFFIRM Online found this visual approach useful to disseminate important information to LGBTQ+ youth, and youth found it reassuring as many reported a lack of focus due to the pandemic.

\section{Therapeutic Engagement: Demonstrate an LGBTQ+ Affirming Stance Online}

As social work clinicians, it is critical to affirm the identities of LGBTQ+ youth while ensuring that there is an appreciation and acknowledgment of intersectional anti- LGBTQ+ stigma and discrimination in their lives (Craig et al. 2013; Langdridge 2007). In an online environment, maintaining an affirming stance may require additional steps to ensure that LGBTQ+ youth experience the intended affirmation. For instance, the authors ensured that they increased and repeated their verbal expressions of LGBTQ+ affirmation throughout each session. Affirmation was expressed by making frequent mention of the links between systemic barriers the participants had experienced in relation to their mental health, such as how experiences of homo/bi/transphobia resulted in fear and distrust in public situations. Additionally, to convey LGBTQ+ affirmation, the use of visual tools, e.g., the Zoom whiteboard and screen sharing function to display affirming messages from the AFFIRM workbook, was particularly effective in enhancing LGBTQ+ affirmation in a virtual environment. Extra attention was also placed during session check ins and check outs to enhance the sense of affirmation of participants' intersectional identities. Facilitators acknowledged that members' experiences of racism, ablism, ageism, and xenophobia interacted within and outside of the LGBTQ+ community to impact their wellbeing. For example, group members' stressful experiences, such as being quarantined with non-affirming parents, were supported and further contextualized within a system of oppression by facilitators during check ins. Members were later able to demonstrate an ability to recognize and externalize their experiences of discrimination, such as difficulty developing trust, which was validated by other group members. These strategies facilitated a sense of affirmation among participants.

\section{Engage Cognitive Restructuring Approaches Virtually}

CBT utilizes cognitive strategies (Beck and Alford 2009) to support clients in challenging negative thoughts and beliefs. AFFIRM utilizes the ABCD method and thought stopping for cognitive restructuring among LGBTQ+ youth. In AFFIRM offline, clinicians help LGBTQ+ youth systematically question the "helpfulness" or "utility" of their thought or belief (e.g., "how does the belief that you will be kicked out of your home if you come out serve you?") rather than whether it is "rational" or "dysfunctional" (e.g., "could the belief that you will be kicked out of your home if you come out be irrational or dysfunctional?") (Craig and Austin 2016). This de-stigmatizing reframing of Socratic questioning can help avoid the intensifying of worries and anxiety, while validating the real environmental problems 
(e.g., bullying, familial and peer rejection, homelessness) encountered by LGBTQ+ youth.

In AFFIRM Online, clinicians must engage youth to identify the relationship between cognitions, behaviors and emotions and learn cognitive restructuring in a virtual group setting. To teach these skills, the authors created an electronic fillable AFFIRM workbook in which LGBTQ+ youth could complete thought records and $\mathrm{ABCD}$ worksheets directly on their computers or electronic mobile devices. As many LGBTQ+ youth utilize mobile phones to access the internet (McInroy et al. 2019), it was important that the workbook be mobile-friendly and accessible. Compared to offline groups, clinicians found that they needed to provide more CBT visuals (e.g., CBT triangle on the share screen), $\mathrm{ABCD}$ and thought stopping examples, while also increasing practice time (e.g., providing extra individual reflection time) to better support youth in mastering these skills. Additionally, clinicians needed to better embed cognitive strategies, such as engaging in deliberative reframing and referring back to the ABCD method in later sessions, with practice into each session (including the more behaviorally-focused sessions).

Another useful approach to enable youth to better grasp cognitive skills online was to make use of the group chat on Zoom to share cognitive restructuring (e.g., disputing beliefs) outcomes. This was done through one facilitator acting as a 'note taker', to capture thinking traps (i.e., cognitive distortions) expressed by the participants, while the other facilitator led the group members through the activity. Through discussion, the participants were able to type and submit various reframing statements to be viewed by other group members. Additionally, cognitive restructuring skills were strengthened among LGBTQ+ youth in a virtual environment by having clinicians provide multiple examples of various concepts. For example, when introducing thinking traps (i.e., cognitive distortions), we first provided at least four examples (e.g., "There is something wrong with me because I am pansexual"), before guiding participants through identifying their own thinking traps and subsequent thought record. Facilitators also ensured that participants were allotted enough time to follow up with their fellow group members during online debriefing segments. Members were encouraged to share their thoughts and feelings about their individual work with the larger group, to elicit feedback and support in acquiring new cognitive coping skills. Finally, an overarching approach that supported the acquisition of cognitive restructuring skills online included brief between-session individual support for youth that continued to struggle to understand and apply the skills. This was achieved through email and text contact, in which additional examples, journal writing assignments, and brief phone coaching were provided to support cognitive coping skills acquisition. These methods were then incorporated into the group sessions by facilitators and youth.

\section{Develop Behavioral Strategies Online}

Behavioral skills and strategies may include approaches to modify unhelpful cognitions through activities, actions and problem solving (Webb et al. 2017). In AFFIRM offline, clinicians move through various behavioral experiments and activation, as well as goal setting and contracting related to LGBTQ+ affirming activities (Craig and Austin 2016). AFFIRM spends a considerable amount of time addressing barriers to affirming behaviors, particularly those related to prejudice and discrimination. During the COVID-19 pandemic, LGBTQ+ youth are largely isolating at home. Social distancing may have resulted in the exacerbation of existing mental health and psychosocial problems (Human Rights Campaign Foundation 2020). Telehealth provides a unique opportunity for social work clinicians to intervene when youth are isolated (e.g., socially, geographically, psychologically) to develop behavioral coping skills and tangible problem solving skills to support their wellbeing and navigate their environment. AFFIRM Online has adapted classic behavioral skills to develop supportive activities within LGBTQ+ youth's socially isolated environment. For instance, clinicians have taken extra time to explore virtual or socially distanced activities that would specifically benefit the group members. Behavioral activation activities included joining online communities and events that are LGBTQ+ focused (e.g., fandom communities), and modifying activities (e.g., exercise, social time, sports) that could be conducted individually or with safe social distancing.

In addition, adapting behavioral experiments (i.e., testing and challenging unhelpful cognitions through specific actions) to an online environment can be implemented through online platforms. In working with LGBTQ+ youth, a social work clinician may develop an exposure or experiment through the use of video (e.g., FaceTime, WhatsApp, Google Hangouts) or verbal messages. For example, clinicians can work with youth to collaboratively test out the belief "I'm boring" by setting up a group conversation with friends and intentionally saying things that are "boring" to see how their friends respond. They may also attempt to evaluate further automatic thoughts that arise as they test out their beliefs through behavioral experiments. In the spirit of collaboration (Beck and Alford 2009), clinicians can work together with youth online to ensure predictions are observable and generalize their learning (e.g., "what does this exposure tell you about your fear of being boring to your friends and more generally?") (Oxford Centre for Anxiety Disorders and Trauma 2020). Ultimately, the online adaptation of affirmative CBT facilitated bringing diverse youth together to allow for a sharing of collective online resources and activities that support LGBTQ+ youth mental health. 


\section{Developing an LGBTQ+ Affirming Online Social Support Network}

An essential component of affirmative CBT offline is developing LGBTQ+ youths' affirming social support network. Given that LGBTQ+ youth often experience a lack of safety (Craig and McInroy 2014) as well as peer rejection and social isolation offline (Taylor et al. 2011), many have chosen to foster a sense of community and friendships online (McInroy and Craig 2018). Therefore, AFFIRM Online further leverages LGBTQ+ youth's strengths to form online communities by creating an online environment in which youth can share resources, connect to online social spaces, and form bonds with other group members. An essential adaptation for AFFIRM Online involves assessing existing youth support networks as well as building a sense of community among AFFIRM members early in the intervention. While this process may occur in later stages of the offline AFFIRM intervention, it is crucial that social work clinicians proactively support LGBTQ+ youth in supporting one another online. For instance, during the early sessions of the AFFIRM Online group, some youth have formed social media groups to continue connecting between sessions and post-intervention. The proactive encouragement of clinicians for LGBTQ+ youth to safely share their contact information (e.g., Instagram, Snapchat, or TikTok usernames) can help facilitate those connections.

\section{Clinical Case Study}

The following case study based on an actual AFFIRM Online group session will be used to demonstrate a "reallife" telehealth application of CBT clinical skills. This examples focuses on an LGBTQ+ youth named "Jade". The authors obtained permission from the youth (including a suggested pseudonym to protect confidentiality).

Jade is 17-years-old, identifies as gender non-binary, pansexual, Indigenous, and uses they/them pronouns. Jade joined AFFIRM Online because they were experiencing increased stress, anxiety, and depression due to social distancing, not being out to family (living with mother, father, and younger brother) and quarantining with them, and not being able to see the few friends they have at school due to the physical closure of schools. Jade also struggles with accepting their gender identity and lives with significant worry and anxiety that if they came out to their friends and family they would be rejected. As Jade is not out to their parents yet recognized their own declining mental health, they sought out supports online and completed the group consent/assent forms while working closely with the group facilitator. Given the lack of family involvement, the facilitator checked the current legal restrictions and noted that youth over age 16 were able to receive up to two months of care without parental permission. Given that many parents reject or abuse their children with minority sexual or gender identities, legal and safety issues must be identified to avoid unintentionally outing adolescents or putting them at further risk in their homes (McInroy 2016). Jade, living in a remote rural community, was able to join an online AFFIRM group with eight other LGBTQ+ youth. Previous attempts by Jade to seek out counselling for their mental health had been thwarted in the past due to their social anxiety, however the online platform reduced barriers for Jade to receive therapy and reduced their anxiety about leaving their home to seek support at a mental health agency. Since there were no LGBTQ+ specific services open and available in their community, AFFIRM Online was an important opportunity for Jade to develop mental health coping skills, be affirmed in their intersectional identities, and develop social supports with other youth.

AFFIRM dedicates Sessions 3 and 4 to learning cognitive restructuring skills. The following excerpt between a social work clinician and Jade during an AFFIRM group highlights the online adaptation to teach these skills to Jade and the other group members through an initial individualized focus on Jade's experiences. Jade, who volunteered to provide an example of their automatic thoughts and work through the ABCD method with the clinician, struggled with significant cognitions (referred to as hot thoughts) such as "no one will ever accept my gender identity"; "my friends will hate me if I come out to them"; "my parents will disown me"; "I'll never be happy because of who I am." These frequent thoughts were fuelled by underlying core beliefs, "I am unworthy" and "I am unlovable," which were identified in previous sessions.

\section{AFFIRM Online Group Session 4: Adapting our Thoughts and Behaviors}

Social Work Clinician: Let's do a screen share of the CBT triangle to review the connection between thoughts, feelings and behaviors. Can everyone see the CBT triangle on the screen? I will describe the CBT triangle for the group.

Group: Yes.

Social Work Clinician: Jade, thanks for volunteering to share your experiences with all of us, and some of the hot thoughts you were having last night.

Jade: Sure. I'm going to type them in the group chat because I'm a little nervous talking about them and I don't want my mom to overhear them.

Social Work Clinician: Not a problem! The group chat is a good alternative to contributing verbally in the group. So, maybe you can share some of the thoughts you were having 
last night that were stressing you out? (uses Zoom pointer to signal the "thoughts" circle on the CBT triangle).

Jade: (Typing in the group chat). No one will ever accept me as non-binary and pan. All my friends and family will hate me and I'll never be happy. I'm doomed!

Social Work Clinician: That sounds very painful. It is a true testament to your strength and courage that you continue to face the world despite regularly having these painful thoughts. Can others relate to some of these thoughts?

Group: Members nodding, audible yes' from the group, and group members typing in supportive statements (e.g., "I totally get that, me too!") to Jade.

Jade: (Speaks audibly) It's the worst!

Social Work Clinician: I too have grappled with similar thoughts as a youth and young adult. It can be very hard to have all these thoughts swirling around. Have others ever felt similarly? (Participants nod or say yes). What type of feelings and emotions do you feel when you think of these thoughts? (uses Zoom pointer to signal the "feelings" circle on the CBT triangle). You can take a look at the Emotions worksheet in our Workbooks to better identify the associated feelings (scrolls to Emotions worksheet in screen share mode).

Jade: These thoughts make me feel scared, anxious, angry, and sad.

Social Work Clinician: That makes complete sense to me and thank you for sharing. Let's start putting these responses in the ABCD Method worksheet (scrolls to worksheet in screen share mode). So, the activating event was that you were lying in bed and your mind was racing with painful thoughts (types in activating event in the A quadrant of worksheet). Then, we can fill out the beliefs and thoughts you were having (types in Jade's identified hot thoughts). Jade, for quadrant C (consequences), we can put the emotions you had, but what other types of consequences resulted due to the hot thoughts?

Jade: I started to get fast breathing, felt hot and sweaty. I shot out of bed and smoked some of my weed vape to calm me. I don't think that's the best way to deal with this because then I can't sleep when I'm high. [a group member types in the chat that they also can't sleep after vaping].

Social Work Clinician: I totally get that. It sounds like the coping behavior you tried, smoking weed, doesn't necessarily work with these anxious thoughts and beliefs. Okay, let's type that into quadrant C (types in Jade's response). So, as we move to quadrant $\mathrm{D}$, here we can start disputing or "talking back" to these hot thoughts. How do you think you could talk back to these painful beliefs?

Jade: I'm not sure, I'm stuck. They seem pretty real when I'm anxious. I don't know what to say.

Social Work Clinician: That's totally normal. I sometimes get stuck as well when I have anxious thoughts and believe them to be $100 \%$ true. It will be important for us to consider what thoughts are helpful here and what are unhelpful. Are there advantages and disadvantages to these thoughts?

Jade: Well, if I think about them a lot, I can figure out how to deal with all this stuff. But they also get really intense, make me feel like crap and I end up in the worst mood. And then I have no one to talk to isolating at home.

Social Work Clinician: That's really tough. Okay, so in many ways these thoughts aren't serving you well.

Jade: No (looks despondent, shoulders sag and eyes look down).

Social Work Clinician: That's good insight that you have though. A helpful approach when we can't think of how to dispute or debate our thoughts and beliefs is to ask: "what would a trusted person in my life say about these beliefs?", or "what would my queer and trans friends, including in this group, say about these beliefs?".

Jade: Yeah, I don't know.

Social Work Clinician: Would it be okay to ask the group if they might have any responses to dispute the beliefs and replace them with more supportive ones?

Jade: Sure!

Social Work Clinician: Okay, so, feel free to type your responses in the group chat or unmute yourself and signal that you'd like to speak (many youth type supportive beliefs to dispute Jade's hot thoughts, some speak directly to Jade). Jade, which of all these new beliefs seem most believable to you?

(The group observes Jade reading the responses in the chat, identifying which responses resonated for them before responding).

Jade: I think I could replace these thoughts with: "I am out to some queer and trans friends online and they accept and love me"; "There are people who will accept me if I come out, I just need to be careful with who I come out to right now"; "I can be happy as a non-binary and pan person." I think these are nice thoughts. I could maybe try to shift the idea that "I'm doomed" with these new thoughts and "I am not doomed, I have love and support around me."

Social Work Clinician: That makes a lot of good sense! [other group members give thumbs up and use Zoom emojis to support Jade]. Great work, Jade! When you say these new thoughts aloud how does it feel?

Jade: I feel better, calmer, more hopeful.

Social Work Clinician: That's awesome. Would you be able to practice using those new beliefs when you're experiencing the negative hot thoughts again at home?

Jade: I think so.

Social Work Clinician: I can copy and paste your and the group's dispute responses and send it to you in an electronic document.

Jade: Cool, thanks. 
Social Work Clinician: We can follow up and talk about how that worked for you next week. I want to thank you for being brave enough to share your experiences with me and the group - your openness helps us all understand how we can think about things differently and realize how we are all working on this together!

\section{Discussion}

As demonstrated by the case study and the adaptation processes, critical clinical skills can be tailored to a telehealth platform to benefit marginalized populations. The clinician utilized the technology-mediated group structure and Zoom functions (group chat, share screen, white board) to support Jade in walking through the ABCD method worksheet and developing cognitive restructuring skills. The clinician's validation of Jade's struggles and their effective use of self in their own struggle with negative beliefs all support Jade in moving through the activity. In addition, the clinician harnessed the power of the group to evoke thought disputation and to elicit social support for Jade; this strategy is very effective offline and can be implemented online through the use of group chat. Further, the clinician has the advantage of generating a supportive electronic document that Jade can retrieve on their mobile phone to help consolidate the new beliefs generated during the session and continue practicing this new skill. The social work clinician supported Jade to problem solve and develop a safe social network by relating their sharing to coming out to safer friends, finding healthier coping behaviors when experiencing stress and anxiety, and teaching grounding and cognitive restructuring skills when triggered by negative thoughts. Jade ended up increasingly coming out to others over the course of the intervention, engaged in online behavioral experiments to further test out negative beliefs, and started attending online LGBTQ+ events with other AFFIRM Online youth. The case study and recommendations aligning with extant literature on online CBT interventions for youth (Grist et al. 2019; Khanna et al. 2007), illustrate the potential for social work clinicians to adapt their offline clinical interventions into an online environment, either within a group or individual format.

\section{Limitations and Conclusion}

While telehealth is timely and may have been useful for LGBTQ+ youth during the COVID-19 pandemic, there are some limitations to consider. Privacy and confidentiality may be more difficult to manage online (Reamer
2014) and a telehealth intervention may not be appropriate for all youth or clinical situations. For instance, for youth experiencing abuse, instability, or extreme lack of safety at home, participation in an online group from their home may increase risks. Additionally, youth may not feel comfortable using Zoom or other similar platforms and may self-select out of the group. Clinicians should support those youth who self-select out of the group, or who are unable to participate, by connecting them to alternative supports and resources.

Given that the stressors experienced by LGBTQ+ youth suggest that telehealth has potential to mitigate negative mental health risks (EGALE 2020; Whittington et al. 2020), adapting an empirically-supported CBT intervention such as AFFIRM to a virtual online environment is an important step towards increasing access to clinical services. Telehealth interventions can support vulnerable populations, such as LGBTQ+ youth, to deal with stressful situations and obtain critical guidance from affirming communities, even if they are unable to modify their current offline environments. This paper illustrates multiple applications of affirmative CBT skills to telehealth with LGBTQ+ youth and suggests clinical strategies to support clients to develop crucial coping skills during challenging times.

Acknowledgements We are grateful to the AFFIRM Online participants, to the inspiring clinical facilitators, and to Andrew Eaton, Vivian Leung and Nelson Pang for their work as members of the AFFIRM team. We thank Cheryl Dobinson and Planned Parenthood Toronto for their partnership. Dr. Craig is the Canada Research Chair in Sexual and Gender Minority Youth.

Funding This study was funded by the Social Sciences and Humanities Research Council of Canada through a Partnership Grant (SSHRC \#895-2018-1000) and by the Public Health Agency of Canada through their Community Action Fund (PHAC \#1718-HQ-000697).

\section{Compliance with Ethical Standards}

Conflict of interest The authors declare that they have no conflicts of interest.

Ethical Approval This study was approved by the University of Toronto's Health Science Research Ethics Board (Protocol ID\# 35229).

\section{References}

Andrews, G., Cuijpers, P., Craske, M. G., McEvoy, P., \& Titov, N. (2010). Computer therapy for the anxiety and depressive disorders is effective, acceptable and practical health care: A metaanalysis. PLoS ONE, 5(10), e13196. https://doi.org/10.1371/journ al.pone.0013196.

American Psychological Association (APA). (2013). Guidelines for the practice of telepsychology. https://www.apa.org/practice/guide lines/telepsychology. 
Austin, A., \& Craig, S. L. (2015). Empirically supported interventions for sexual and gender minority youth. Journal of Evidence-Based Social Work, 12(6), 567-578.

Backhaus, A., Agha, Z., Maglione, M. L., Repp, A., Ross, B., Zuest, D., et al. (2012). Videoconferencing psychotherapy: A systematic review. Psychological Services, 9(2), 111-131. https://doi. org/10.1037/a0027924.

Beck, A. T. (1993). Cognitive therapy: Past, present, and future. Journal of Consulting and Clinical Psychology, 61(2), 194-198. https ://doi.org/10.1037//0022-006x.61.2.194.

Beck, A. T., \& Alford, B. (2009). Depression: Causes and treatment. Philadelphia: University of Pennsylvania Press.

Bochicchio, L., Reeder, K., Ivanoff, A., Pope, H., \& Stefancic, A. (2020). Psychotherapeutic interventions for LGBTQ+ youth: a systematic review. Journal of LGBT youth, 1-28.

Borgogna, N. C., McDermott, R. C., Aita, S. L., \& Kridel, M. M. (2019). Anxiety and depression across gender and sexual minorities: Implications for transgender, gender nonconforming, pansexual, demisexual, asexual, queer, and questioning individuals. Psychology of Sexual Orientation and Gender Diversity, 6(1), 54-63. https://doi.org/10.1037/sgd0000306.

Burton, C. M., Marshal, M. P., Chisolm, D. J., Sucato, G. S., \& Friedman, M. S. (2013). Sexual minority-related victimization as a mediator of health disparities in sexual minority youth: A longitudinal analysis. Journal of Youth and Adolescence, 42(3), 394-402. https://doi.org/10.1007/s10964-012-9901-5.

Camhi, S. S., Herweck, A., \& Perone, H. (2020). Telehealth training is essential to care for underserved populations: A medical student perspective. Medical science educator, 30(3), 1287-1290. https ://doi.org/10.1007/s40670-020-01008-w.

Canadian Psychological Association (CPA). (2006). Ethical guidelines for psychologists providing psychological services via electronic media. http://www.cpa.ca/aboutcpa/committees/ethics/psychservi ceselectronically/

Compton, S. N., March, J. S., Brent, D., Albano, A. M., Weersing, R., \& Curry, J. (2004). Cognitive-behavioral psychotherapy for anxiety and depressive disorders in children and adolescents: An evidence-based medicine review. Journal of the American Academy of Child \& Adolescent Psychiatry, 43(8), 930-959. https:// doi.org/10.1097/01.chi.0000127589.57468.bf.

Craig, S. L., \& Austin, A. (2016). The AFFIRM open pilot feasibility study: A brief affirmative cognitive behavioral coping skills group intervention for sexual and gender minority youth. Children and Youth Services Review, 64, 136-144.

Craig, S. L., Austin, A., \& Alessi, E. (2013). Gay affirmative cognitive behavioral therapy for sexual minority youth: A clinical adaptation. Clinical Social Work Journal, 41(3), 258-266.

Craig, S. L., Iacono, G., Austin, A., Eaton, A. D., Pang, N., Leung, V. W., \& Frey, C. J. (2020). The role of facilitator training in intervention delivery: Preparing clinicians to deliver AFFIRMative group cognitive behavioral therapy to sexual and gender minority youth. Journal of Gay \& Lesbian Social Services, 1-22.

Craig, S. L, Leung, V., Pascoe, R., Pang, N., Iacono, G., Austin, A. \& Dillon, F. (2021). AFFIRM Online: Utilizing an affirmative cognitive-behavioural digital intervention to improve mental health, access and engagement among LGBTQA+ youth and young adults. International Journal of Environmental Research and Public Health, 18(4), 1541. https://doi.org/10.3390/ijerph18041541.

Craig, S. L., \& McInroy, L. (2014). You can form a part of yourself online: The influence of new media on identity development and coming out for LGBTQ youth. Journal of Gay and Lesbian Mental Health, 18(1), 95-109. https://doi.org/10.1080/19359 705.2013.777007.

Craig, S. L., McInroy, L., Austin, A., Smith, M., \& Engle, B. (2012). Promoting self-efficacy and self-esteem for multiethnic sexual minority youth: An evidence-informed intervention. Journal of Social Service Research, 38(5), 688-698.

Craig, S. L., McInroy, L. B., D’Souza, S. A., Austin, A., McCready, L. T., Eaton, A. D., et al. (2017). Influence of information and communication technologies on the resilience and coping of sexual and gender minority youth in the United States and Canada (Project\# Queery): Mixed methods survey. JMIR Research Protocols, 6(9), e189.

Craig, S. L., McInroy, L. B., Eaton, A. D., Iacono, G., Leung, V. W., Austin, A., \& Dobinson, C. (2019). An affirmative coping skills intervention to improve the mental and sexual health of sexual and gender minority youth (Project Youth AFFIRM): protocol for an implementation study. JMIR Research Protocols, 8(6), e13462.

Craig, S. L., McInroy, L. B., McCready, L. T., Di Cesare, D. M., \& Pettaway, L. D. (2015). Connecting without fear: Clinical implications of the consumption of information and communication technologies by sexual minority youth and young adults. Clinical Social Work Journal, 43(2), 159-168.

Davies, E. B., Morriss, R., \& Glazebrook, C. (2014). Computer-delivered and web-based interventions to improve depression, anxiety, and psychological well-being of university students: A systematic review and meta-analysis. Journal of Medical Internet Research, 16(5), e130. https://doi.org/10.2196/jmir.3142.

Egale. (2020, April 6). Impact of COVID-19: Canada's LGBTQI2S community in focus. https://egale.ca/wp-content/uploads/2020/04/ Impact-of-COVID-19-Canada\%E2\%80\%99s-LGBTQI2S-Commu nity-in-Focus-2020-04-06.pdf

Fish, J. N., McInroy, L. B., Paceley, M. S., Williams, N. D., Henderson, S., Levine, D. S., \& Edsall, R. N. (2020). "I'm Kinda Stuck at home with unsupportive parents right now": LGBTQ youths' experiences with COVID-19 and the importance of online support. Journal of Adolescent Health, 67(3), 450-452. https://doi. org/10.1016/j.jadohealth.2020.06.002.

Fowlkes, A. (2020, April 25). COVID-19: The gay, the homeless, the gay and homeless. Forbes. https://www.forbes.com/sites/ashle efowlkes/2020/04/25/covid-19-the-gay-the-homeless-the-gayand-homeless/\#5f3baa634ad7

Fleming, T., Dixon, R., Frampton, C., \& Merry, S. (2012). A pragmatic randomized controlled trial of computerized CBT (SPARX) for symptoms of depression among adolescents excluded from mainstream education. Behavioural and Cognitive Psychotherapy, 40(5), 529-541. https://doi.org/10.1017/S1352465811000695.

Furmark, T., Carlbring, P., Hedman, E., Sonnenstein, A., Clevberger, P., Bohman, B., et al. (2009). Guided and unguided self-help for social anxiety disorder: Randomized controlled trial. The British Journal of Psychiatry, 195(5), 440-447. https://doi.org/10.1192/ bjp.bp.108.060996.

Gibb, J. K., DuBois, L. Z., Williams, S., McKerracher, L., Juster, R.-P., \& Fields, J. (2020). Sexual and gender minority health vulnerabilities during the COVID-19 health crisis. American Journal of Human Biology, 32, e23499. https://doi.org/10.1002/ajhb.23499.

Grist, R., Croker, A., Denne, M., \& Stallard, P. (2019). Technology delivered interventions for depression and anxiety in children and adolescents: A systematic review and meta-analysis. Clinical Child and Family Psychology Review, 22(2), 147-171. https ://doi.org/10.1007/s10567-018-0271-8.

Hatzenbuehler, M. L. (2009). How does sexual minority stigma "get under the skin"? A psychological mediation framework. Psychological Bulletin, 135(5), 707-730. https://doi.org/10.1037/a0016 441.

Hatzenbuehler, M. L., McLaughlin, K. A., \& Nolen-Hoeksema, S. (2008). Emotion regulation and internalizing symptoms in a longitudinal study of sexual minority and heterosexual adolescents. Journal of Child Psychology and Psychiatry, 49(12), 1270-1278. https://doi.org/10.1111/j.1469-7610.2008.01924.x. 
Hightow-Weidman, L. B., Muessig, K. E., Pike, E. C., LeGrand, S., Baltierra, N., Rucker, A. J., \& Wilson, P. (2015). HealthMpowerment org: Building community through a mobile-optimized, online health promotion intervention. Health Education \& Behavior, 42(4), 493-499.

Hollis, C., Falconer, C. J., Martin, J. L., Whittington, C., Stockton, S., Glazebrook, C., \& Davies, E. B. (2017). Annual Research Review: Digital health interventions for children and young people with mental health problems-a systematic and meta-review. Journal of Child Psychology and Psychiatry, 58(4), 474-503. https://doi. org/10.1111/jcpp.12663.

Human Rights Campaign Foundation. (2020). The economic impact of COVID-19 on the LGBTQ community. https://assets2.hrc.org/files /assets/resources/COVID19-EconomicImpact-IssueBrief-04222 0.pdf?_ga $=2.175101600 .38948014 .1588184139-1016871562$ .1547937697

Iacovides, I., Samra, R., Lucassen, M., \& Wallace, L. (2017). Gamebased cognitive behavioural therapy (CBT) for sexual minority youth: Rainbow SPARX in the UK. Chi Play.

Kaiser Family Foundation (2020, November 15). Opportunities and barriers for telemedicine in the U.S. during the COVID-19 emergency and beyond. Women's Health Policy. https://www.kff. org/womens-health-policy/issue-brief/opportunities-and-barri ers-for-telemedicine-in-the-u-s-during-the-covid-19-emergencyand-beyond/

Kahn, J. M. (2015). Virtual visits: Confronting the challenges of telemedicine. The New England Journal of Medicine, 372(18), 1684-1685. https://doi.org/10.1056/nejmp1500533.

Khanna, M., Aschenbrand, S. G., \& Kendall, P. C. (2007). New frontiers: Computer technology in the treatment of anxious youth. The Behavior Therapist, 30(1), 22-25.

Kiropoulos, L. A., Klein, B., Austin, D. W., Gilson, K., Pier, C., Mitchell, J., \& Ciechomski, L. (2008). Is internet-based CBT for panic disorder and agoraphobia as effective as face-to-face CBT? Journal of anxiety disorders, 22(8), 1273-1284. https:// doi.org/10.1016/j.janxdis.2008.01.008.

Langdridge, D. (2007). Gay affirmative therapy: A theoretical framework and defense. Journal of Gay and Lesbian Psychotherapy, 11(1-2), 27-43. https://doi.org/10.1300/J236v11n01_03.

Lucassen, M. F., Merry, S. N., Hatcher, S., \& Frampton, C. M. (2015). Rainbow SPARX: A novel approach to addressing depression in sexual minority youth. Cognitive and Behavioral Practice, 22(2), 203-216.

McCarty, D., \& Clancy, C. (2002). Telehealth: Implications for social work practice. Social Work, 47(2), 153-161. https://doi. org/10.1093/sw/47.2.153.

McInroy, L. B. (2016). Pitfalls, potentials, and ethics of online survey research: LGBTQ and other marginalized and hard-toaccess youths. Social Work Research, 40(2), 83-94. https://doi. org/10.1093/swr/svw005.

McInroy, L. B., \& Craig, S. L. (2018). Online fandom, identity milestones, and self-identification of sexual/gender minority youth. Journal of LGBT Youth, 15(3), 179-196.

McInroy, L. B., McCloskey, R. J., Craig, S. L., \& Eaton, A. D. (2019). LGBTQ+ youths' community engagement and resource seeking online versus offline. Journal of Technology in Human Services, $37(4), 315-333$

Merrill, K. A., Tolbert, V. E., \& Wade, W. A. (2003). Effectiveness of cognitive therapy for depression in a community mental health center: A benchmarking study. Journal of Consulting and Clinical Psychology, 71(2), 404-409. https://doi. org/10.1037/0022-006x.71.2.404.

Meyer, I. H. (2003). Minority stress and mental health in gay men. In L. D. Garnets \& D. C. Kimmel (Eds.), Psychological perspectives on lesbian, gay, and bisexual experiences (pp. 699-731). New York: Columbia University Press.
National Association of Social Workers (NASW). (n.d.). Telehealth. https://www.socialworkers.org/Practice/Infectious-Diseases/ Coronavirus/Telehealth.

Oxford Centre for Anxiety Disorders and Trauma. (2020). Cognitive therapy for social anxiety disorder (CT-SAD): Guidance for conducting treatment remotely during the coronavirus pandemic (Version 1).।

Poppelaars, M., Tak, Y. R., Lichtwarck-Aschoff, A., Engels, R. C. M. E., Lobel, A., Merry, S. N., et al. (2016). A randomized controlled trial comparing two cognitive-behavioral programs for adolescent girls with subclinical depression: A school-based program (Op Volle Kracht) and a computerized program (SPARX). Behaviour Research and Therapy, 80, 33-42. https://doi.org/10.1016/j. brat.2016.03.005.

Reamer, F. G. (2014). Clinical social work in a digital environment: Ethical and risk-management challenges. Clinical Social Work Journal, 43(2), 120-132. https://doi.org/10.1007/s1061 5-014-0495-0.

Russell, S. T., \& Fish, J. N. (2016). Mental health in lesbian, gay, bisexual, and transgender (LGBT) youth. Annual Review of Clinical Psychology, 12, 465-487. https://doi.org/10.1146/annurev-clinp sy-021815-093153.

Saidipour, P. (2020). The precedent of good enough therapy during unprecedented times. Clinical Social Work Journal. https://doi. org/10.1007/s10615-020-00776-7.

Scott, K., Klech, D., Lewis, C. C., \& Simons, A. D. (2016). What did they learn? Effects of a brief cognitive behavioral therapy workshop on community therapists' knowledge. Community mental health journal, 52(8), 998-1003. https://doi.org/10.1007/s1059 7-015-9876-2.

Silverman, W. K., Pina, A. A., \& Viswesvaran, C. (2008). Evidencebased psychosocial treatments for phobic and anxiety disorders in children and adolescents. Journal of Clinical Child \& Adolescent Psychology, 37(1), 105-130. https://doi.org/10.1080/1537441070 1817907.

Simons, A. D., Padesky, C. A., Montemarano, J., Lewis, C. C., Murakami, J., Lamb, K., et al. (2010). Training and dissemination of cognitive behavior therapy for depression in adults: A preliminary examination of therapist competence and client outcomes. Journal of Consulting and Clinical Psychology, 78(5), 751-756. https://doi.org/10.1037/a0020569.

Spence, S. H., Donovan, C. L., March, S., Gamble, A., Anderson, R. E., Prosser, S., \& Kenardy, J. (2011). A randomized controlled trial of online versus clinic-based CBT for adolescent anxiety. Journal of Consulting and Clinical Psychology, 79(5), 629-642. https:// doi.org/10.1037/a0024512.

Tasnim, S., Trisha, N. F., Fan, Q., Sultana, A., Mishu, S. H., Hasan, N. T., \& Hossain, M. (2020, February 5). Improving mental health and wellbeing among sexual and gender minorities using digital interventions: A systematic review. https://doi.org/10.31234/osf. io/9wu42

Taylor, C., Peter, T., McMinn, T. L., Elliott, T., Beldom, S., Ferry, A., Gross, Z., Paquin, S., \& Schachter, K. (2011). Every class in every school: The first national climate survey on homophobia, biphobia, and transphobia in Canadian schools. Final report. Toronto, ON: Egale Canada Human Rights Trust. https://egale.ca/aware ness/every-class/

True Colors United. (2020). Our issue. https://truecolorsunited.org/ our-issue/

Webb, C. A., Rosso, I. M., \& Rauch, S. L. (2017). Internet-based cognitive behavioral therapy for depression: Current progress \& future directions. Harvard Review of Psychiatry, 25(3), 114-122. https ://doi.org/10.1097/hrp.0000000000000139.

Whittington, C., Hadfield, K., \& Calderón, C. (2020). The lives and livelihoods of many in the LGBTQ community are at risk amidst COVID-19 crisis. Human Rights Campaign Foundation. https:// 
www.hrc.org/resources/the-lives-and-livelihoods-of-many-in-thelgbtq-community-are-at-risk-amidst-covid-19-crisis

Wright, B., Tindall, L., Littlewood, E., Allgar, V., Abeles, P., Trépel, D., \& Ali, S. (2017). Computerised cognitive-behavioural therapy for depression in adolescents: feasibility results and 4-month outcomes of a UK randomised controlled trial. BMJ Open, 7(1), e012834. https://doi.org/10.1136/bmjopen-2016-012834.

Zhou, X., Snoswell, C. L., Harding, L. E., Bambling, M., Edirippulige, S., Bai, X., \& Smith, A. C. (2020). The role of telehealth in reducing the mental health burden from COVID-19. Telemedicine and e-Health, 26(4), 377-379. https://doi.org/10.1089/tmj.2020.0068.

Publisher's Note Springer Nature remains neutral with regard to jurisdictional claims in published maps and institutional affiliations.
Shelley L. Craig is a full Professor at the Factor-Inwentash Faculty of Social Work at the University of Toronto. She currently holds a Canada Research Chair in Sexual and Gender Minority Youth.

Gio lacono is an Assistant Professor at the School of Social Work at the University of Connecticut.

Rachael Pascoe is a PhD student at the Factor-Inwentash Faculty of Social Work at the University of Toronto.

Ashley Austin is a full Professor at the School of Social Work at Barry University. 